\title{
Assessment of Health and Health Related Problems in the Community of Mettu Town, South Western, Ethiopia, 2021: A Community Based Descriptive Cross Sectional Study
}

\author{
Gudisa Bereda ${ }^{1, *}$, Gemechis Bereda ${ }^{2}$ \\ ${ }^{1}$ Department of Pharmacy, Negelle Health Sciences College, Guji, Ethiopia \\ ${ }^{2}$ Awash Bank, Addis Ababa, Ethiopia
}

Email address:

gudisabareda95@gmail.com (G. Bereda)

${ }^{*}$ Corresponding author

\section{To cite this article:}

Gudisa Bereda, Gemechis Bereda. Assessment of Health and Health Related Problems in the Community of Mettu Town, South Western, Ethiopia, 2021: A Community Based Descriptive Cross Sectional Study. International Journal of Pharmacy and Chemistry.

Vol. 7, No. 3, 2021, pp. 37-44. doi: 10.11648/j.ijpc.20210703.11

Received: May 15, 2021; Accepted: July 5, 2021; Published: July 13, 2021

\begin{abstract}
Background: Community Based Education is an educational philosophy aims at developing professionals with problem identification and solving skills and positive attitudes to serve the society. The communities' health need is mostly rounded on either communicable or non-communicable diseases in which communicable diseases remain the most vital health problems in the developing countries. Objective: The intent of this survey was to ascertain the apprehensive of health and health related problems in the community of Mettu town, south western, Ethiopia. Methods and materials: A community based descriptive cross sectional survey was employed in Mettu town, from January 17/2021-May 25/2021. A stratified random sampling technique was applied, and data was gathered by using an interviewer-administered semi structured questionnaire. Data was analyzed by statistical packages of social sciences 23.0 version statistical software. Findings: The overall prevalence of health related problems was $66.23 \%$. The survey revealed that hypertension $36.94 \%$ and diabetes mellitus $27.8 \%$ were the most common cause of morbidity and mortality. $55.9 \%$ participants had a roof that was made of tin and floor made of cement and $54.79 \%$ of population use pipe water. $68.47 \%$ user of family planning were majorly used $47.13 \%$ injection and $27.39 \%$ of them used implant. $51.59 \%$ women gave birth in health institution and $59.87 \%$ attained ANC services at least one times reguraly. $27.27 \%$ of infants under 6 months are exclusively breastfed and $55.84 \%$ of children start complementary feeding at 6 months. Conclusion and Recommendation: The present survey indicated that the prevalence of health related problems in individuals was escalated dramatically. Majority of Mettu town population was use open field for their waste disposal. Rodent, household flee and bed bug are common insects \& rodents that threaten population health. Health professionals should have to give the population health education about substance, waste disposal, family planning, sanitation condition, and immunization.
\end{abstract}

Keywords: Health, Health Related Problems, Community, Mettu Health Center, Ethiopia

\section{Introduction}

\subsection{Background}

Community based education is a means of achieving educational relevance to community needs and consequently of implementing a community-oriented education program. CBE purposes is; to apply theoretical knowledge in to practice, develop a sense of societal responsibility and accountability, equip students with skill of problem identification, intervention, project preparation and supplementation to solve societal problem, designed in a way that allows students to systematically assess, identify gaps, and analyze community health needs [1]. It is one way of grasping this journey of sustaining the health of a community [2]. 


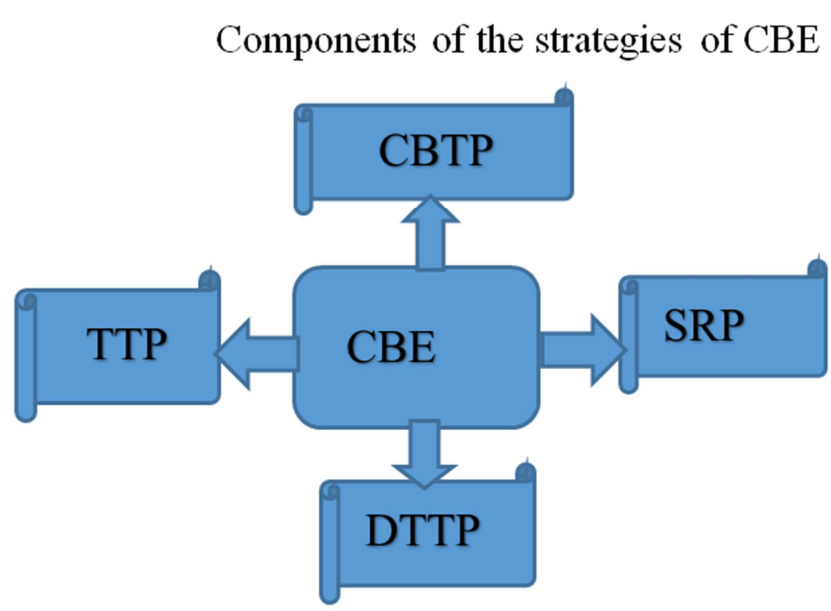

Figure 1. Schematic presentation of the components of CBE strategies.

Sustaining a healthy community is the goal of every part of the world [3]. In Africa, communicable diseases remain the most important health problems. The commonest causes of death and illness in the continent are acute respiratory tract infections, TB, HIV/AIDS/STI, diarrhea disease, malaria and vaccine preventable infections. Epidemic prone diseases such as meningococcal meningitis, cholera, yellow fever, and viral hemorrhagic fevers are also prominent health threats in the country [4]. Ethiopia is one of the developing countries in which most of its population (85\%) mainly depends on agriculture's. Different factors such as lack of professional committeemen, population awareness about the problem of waste disposal, absence of adequate and necessary medical equipment, inaccessible health facility and low health seeking behavior are leading to poor health status of the community. Communicable disease, nutritional problems, maternal and child health problems are the major challenging health care related problems in Ethiopia [5]. In Ethiopia, 60 to $80 \%$ of communicable diseases are attributed to limited access to safe water and inadequate sanitation and hygiene services [6].

\subsection{Statement of the Problem}

Millions of children globally suffer from under nutrition despite many declarations and action plans aimed at combating the phenomenon. In developing countries total of 165 million, 101 million and 51 million children under the age of five are estimated to be stunted, underweight and wasted, respectively [7]. Vector-mediated diseases continue to be a global health threat accounting for an estimated $17 \%$ of all infectious diseases and more than 700,000 deaths. Malaria impact ranks high among all vector borne diseases with a total of 216 million cases and 445,000 deaths [8]. Ethiopian women are at risk of a number of pregnancy complications due to significant socioeconomic disadvantage, poor prior nutrition, being underweight, related conditions such as anemia [9]. The prevalence of NCDs increases throughout the world. It leads to $47 \%$ of the disease burden and $63 \%$ of all mortalities. Of which, $80 \%$ of mortalities occur in developing countries, and the majority of deaths are premature. Further, by the year 2020, global anticipated NCDs burden will rise to $80 \%$ and the majority of deaths $(70 \%)$ will occur in low and middle-income countries [10]. Substance use is a major public health concern that affects every level of society. Individuals, families, communities, and overall government spending are affected by the use psychoactive substances. From those khat, alcohol, tobacco (i.e., cigarettes), and marijuana has the highest prevalence rates across all age groups [11].

\subsection{Significance of the Study}

The program will help us to identify health related problems in the community and give solutions for that problem, and will assist stake holders to be aware of health related problems of the town and consequently take critical measure to improve the health condition of the community. It also guides other interested researchers to conduct more studies in this area to figure out the community health related problems, and also providing health education for the community in different health issues.

\section{Objectives}

\subsection{General Objectives}

To ascertain the health and health related problems of the community in Mettu town, January 2021.

\subsection{Specific Objectives}

1) To identify the main health related problems in Mettu town.

2) To asses' health status of community in Mettu town.

3) To ascertain environmental health and sanitation condition of community.

\section{Methods and Materials}

\subsection{Study Area, Setting and Period}

The study was employed in Mettu town located at $600 \mathrm{~km}$ southwestern of Addis Ababa. The town administratively, consists of 3 kebeles, kebele 1 up to 3 . The town had a total population of 102,074 with sex composition of 50,857 males and 51,217 females. There are 42,496 households in this town with the distribution of 12,743, 14,626, and 15,127 households in each kebele 1 to 3 respectively. In this administration there are 12 governmental schools (1 Kindergarten, 8 primary schools and 3 secondary schools), 14 private schools, 1 governmental referral hospital, 1 non-governmental hospital (Hamlin Fistula Center), 1 governmental health center and 6 health post, 28 private health facilities ( 9 medium clinics, 6 small clinics, 11 Drug stores and 3 pharmacies). There are 30 meditation places, 1 youth center and 1 major market places in this town administration. The study was conducted from January 17/ 2021- May 25/2021. 


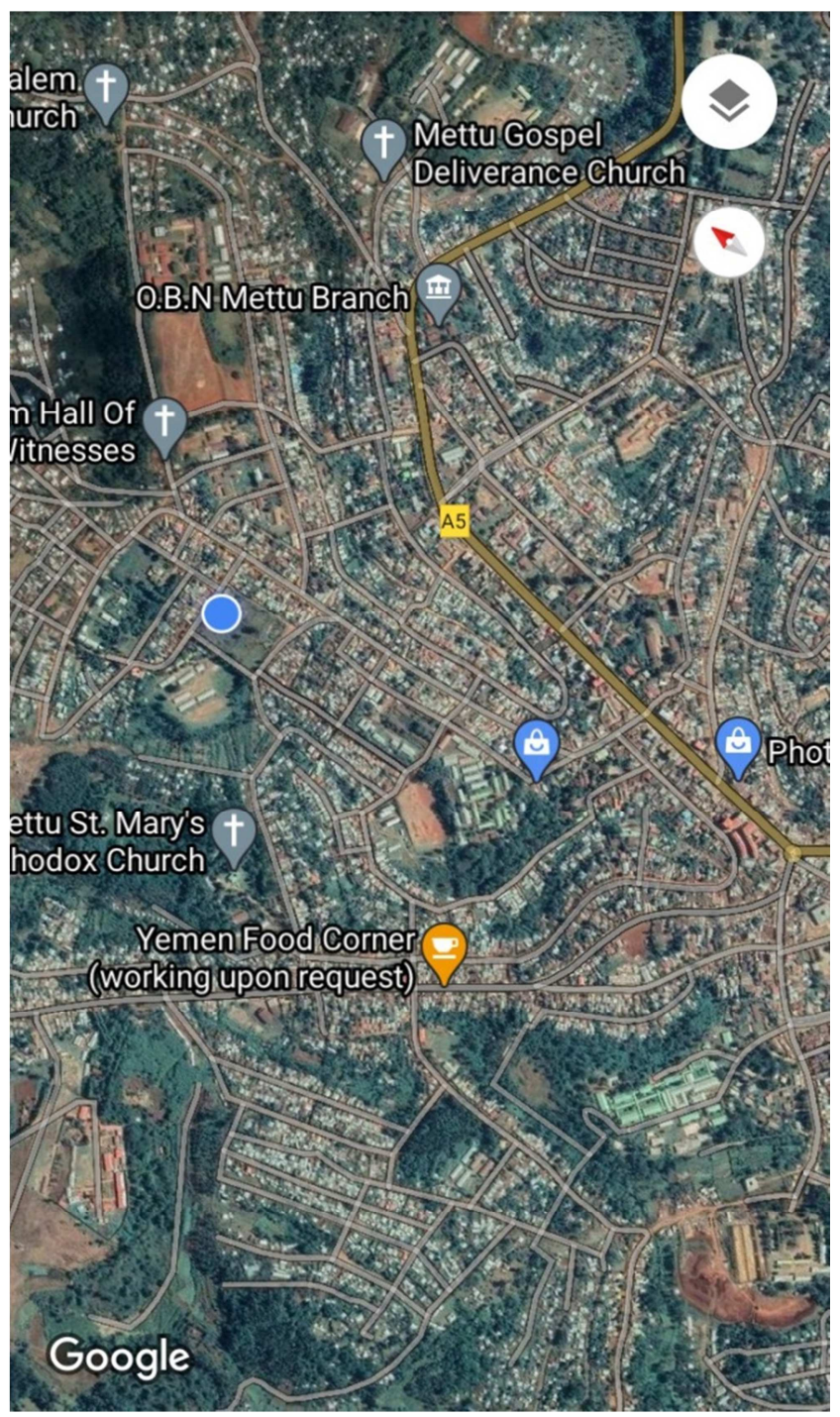

Figure 2. Map of Mettu town, 2021.

\subsection{Study Design}

Community based descriptive cross sectional design was employed.

\subsection{Population}

Source Population: All households of mettu town Target Population: Selected households of mettu town Study unit: The representative of household Sampling unit: House holds

\subsubsection{Inclusion Criteria}

1) All open household of study found in Mettu town during data collection

2) All permanent residents (house hold of study) in mettu town

\subsubsection{Exclusion Criteria}

1) Households in which temporary residents lives in $(<6$ months)

2) Mental illness who was unable to speak \& hear

3) Critically ill

\subsection{Sample Size Determination, Sampling Technique and Procedure}

The sample size was determined by using the Single Population proportion Formula: The sample size was determined based on "P" value which was taken from Bati woreda, oromia zone, Amhara regional state, $\mathrm{P}=0.41$, or $41 \% . n=\frac{(Z a / 2) 2 P(1-P)}{d 2}, \mathrm{n}=(1.96)^{2} 0.41(1-0.41) /(0.05)^{2}=372$. Non response rate $=5 \%$ (to minimize non response rate) $=372+$ $(372 \times 0.05)=19$. So, the total sample size was $=391$.

A stratified random sampling technique was applied to select the study units. The total sample was proportionally allocated for these kebeles based on their total household number using the formula $n j=n \times N j / N$, nj=sample of each kebeles, $\mathrm{Nj}=$ House hold of each kebeles, $\mathrm{n}=$ Sample size, $\mathrm{N}=$ Total house hold of mettu town. There are 42,496 households in this town with the distribution of 12,743, 14,626, and 15,127 households in each kebele 1 to 3 respectively, and then sample unit were taken by using simple random sampling system. Then the required sample size was selected by lottery method.

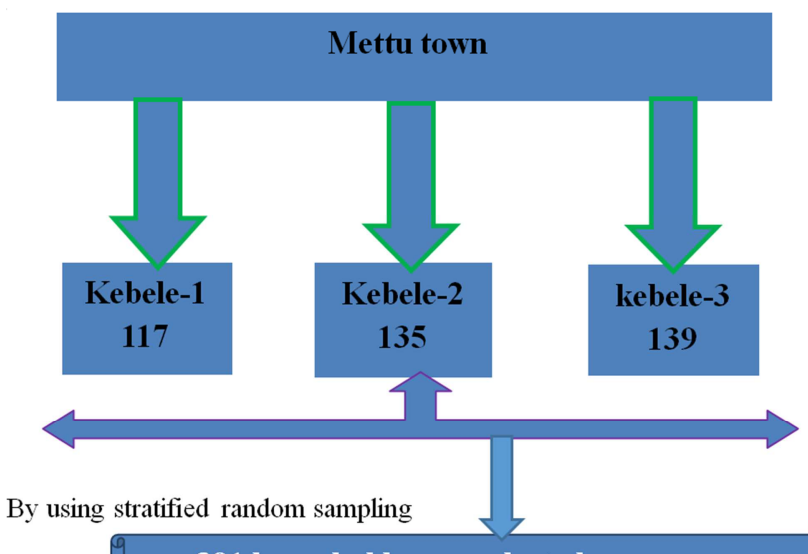

391 houscholds was selected

Figure 3. Schematic presentation of sampling technique in Mettu town, 2021.

\subsection{Study Variables}

Dependent Variables

1) Health condition

2) Hygiene and sanitation problem

3) Maternal and neonatal, child health problem

4) Major communicable or NCDs diseases and morbidity and mortality problem

Independent Variables

Socio-demographic status (age, sex, religion, ethnicity, marital status, monthly income, occupational status, educational status)

\subsection{Data Collection Tools and Quality Control}

The data was gathered by using validated structured questionnaire and an interview. The questionnaire consisted of socio-demographic characteristics, environmental health survey, mother and child health /family planning practice survey, social substance use survey and morbidity and 
mortality survey in individuals from the selected house hold. The data was gathered by using a face to face interview and the respondents were any family member, but we were given a priority for the mother. The study participants were given detail information about what was done and the purpose of the study. We were considering the house hold as a non-respondent if they refuse to participate in the study. The questionnaire was gingerly assessed and pre-tested on $5 \%$ of the sample size which begin to be 20 individuals who dwell in Urumu town prior to the genuine data gathering. In addition to the principal investigator, two supervisors were responsible for observing the data gathering process. The data was collected by 5 data collectors from different departments such as Nursing, Psychiatry, Midwifery, Pharmacy and Public health. The data collectors undertake 2 days of training to become familiar with questionnaire how they undergo data collection procedures. Before data entry, the gathered data gingerly investigated for completeness by the principal investigator.

At the time of data gathering, filled questionnaires were checked for completeness and consistency of information by the principal investigator. We were also check data completeness and consistency during data entry and cleaning with each other.

\subsection{Data Processing and Analysis}

Data entry, cleaning and analysis was carried out by using statistical packages of social sciences 23.0 version statistical software. Descriptive analysis was used to describe the magnitude of health and health related problems in the community. Thus, categorical variables was summarized as numbers and percentages and finally presented by using charts, appropriate diagrams, tables \& graphs.

\subsection{Ethical Consideration}

Ethical approval and letter of support was acquired from community based education office of mettu university college of health sciences. Participants was assured that their participation was totally freewill, their name was not being stated, data were kept behind the scenes and anonymous and it was used only for community diagnosis purpose. After assuring the confidentiality of responses and obtaining verbal informed consent from the study subjects, information was collected by interviewer-administered questionnaire with strict privacy.

\subsection{Operational Definitions}

1) Health status: is an individuals relative level of wellness and illness, taking into account the presence of biological and physiological dysfunction, symptoms and functional impairment.

2) House hold: All people living together in same house.

3) Head of House hold: Is a person with either sex, who is considered to be the head by other member of that house hold.

4) Critically ill: is a life threatening process that, in absence of medical intervention, is expecting to result in mortality or significant morbidity.

5) Maternal and Child Health: Include those who are aged 15-49 year women and those under five years' old children.

6) Live birth: Number of infants born alive during the last 12 months including anyone who were born alive.

\section{Results}

\subsection{Socio-demographic Characteristics}

Our survey showed that there were 1075 total respondents from 391 households. From those 577 (55.67\%) were male and $215(20 \%)$ were under 18 years of old and most age group line with age of 36-64 were 344 (32\%) from both male and female. Individuals who married were $507(47.16 \%)$ and the most dominant religion and ethnicity were protestant 396 (36.84\%) and Oromo 908 (84.46\%) respectively. Majority of the respondents were students $369(34.33 \%)$ and learning elementary schools. $665(61.86 \%)$ of the respondents had got 501-5000 monthly income.

Table 1. Socio demographic characteristics of respondents, Mettu town, 2021.

\begin{tabular}{|c|c|c|c|}
\hline Variables & Category & Frequency (n) & Percent (\%) \\
\hline \multirow{4}{*}{ Age } & $<18$ & 215 & 20.0 \\
\hline & $19-35$ & 327 & 30.4 \\
\hline & $36-64$ & 344 & 32.0 \\
\hline & $>64$ & 189 & 17.6 \\
\hline \multirow{2}{*}{ Sex } & Male & 577 & 55.67 \\
\hline & Female & 498 & 44.33 \\
\hline \multirow{4}{*}{ Marital status } & Single & 279 & 25.95 \\
\hline & Married & 507 & 47.16 \\
\hline & Divorced & 192 & 17.86 \\
\hline & Widowed & 97 & 9.03 \\
\hline \multirow{4}{*}{ Religion } & Protestant & 396 & 36.84 \\
\hline & Muslim & 310 & 28.84 \\
\hline & Orthodox & 352 & 32.74 \\
\hline & Others & 17 & 1.58 \\
\hline \multirow{3}{*}{ Ethnicity } & Oromo & 908 & 84.46 \\
\hline & Amhara & 106 & 9.86 \\
\hline & Others & 61 & 5.68 \\
\hline \multirow{5}{*}{$\begin{array}{l}\text { Occupational } \\
\text { status }\end{array}$} & Merchant & 231 & 21.48 \\
\hline & Government employment & 347 & 32.28 \\
\hline & Farmer & 47 & 4.37 \\
\hline & Student & 369 & 34.33 \\
\hline & Others & 81 & 7.54 \\
\hline \multirow{4}{*}{$\begin{array}{l}\text { Educational } \\
\text { status }\end{array}$} & Unable to read and write & 144 & 13.39 \\
\hline & Elementary & 381 & 35.46 \\
\hline & High school & 325 & 30.23 \\
\hline & College and above & 225 & 20.92 \\
\hline \multirow{3}{*}{$\begin{array}{l}\text { Monthly } \\
\text { income }\end{array}$} & $>500$ & 187 & 17.4 \\
\hline & $501-5000$ & 665 & 61.86 \\
\hline & $>5000$ & 223 & 20.74 \\
\hline
\end{tabular}

\subsection{Community Practice of Substance}

From total 1075 participants majority of individuals had khat addiction $311(28.93 \%)$ followed by cigarette smoking $248(23.06 \%)$ from both male and female. 
Table 2. Community practice of abused substance in respondents, Mettu town, 2021.

\begin{tabular}{lllllll}
\hline \multirow{2}{*}{ Substance category } & Male & & Female & & Total \\
\cline { 2 - 7 } & Frequency & Percent & Frequency & Percent & Frequency & Percent \\
\hline Khat & 216 & 31.68 & 95 & 24.17 & 311 & 28.93 \\
Cigarette & 207 & 30.35 & 41 & 10.44 & 248 & 23.06 \\
Alcohol & 93 & 13.63 & 33 & 8.39 & 126 & 11.72 \\
Other & 65 & 9.53 & 13 & 3.32 & 78 & 7.26 \\
No & 101 & 14.9 & 211 & 53.68 & 312 & 29.03 \\
\hline
\end{tabular}

\subsection{Environmental Health Survey}

Most study respondents 749 (69.67\%) live in their own house, $601(55.9 \%)$ of study participants house were brick /cement, and the individuals who had Pipe in their compound were 589 (54.79\%). 507 (47.16\%) households had use open field for their waste disposal. The majority of respondents 411 $(38.23 \%)$ had rats (rodents) problem in their house.

Table 3. Environmental health survey of participants, Mettu town, 2021.

\begin{tabular}{|c|c|c|c|}
\hline Variables & Category & Frequency (n) & Percent (\%) \\
\hline \multirow{3}{*}{ Owner of the house } & Private & 749 & 69.67 \\
\hline & Rental & 264 & 24.56 \\
\hline & Others & 62 & 5.77 \\
\hline \multirow{3}{*}{ Housing condition } & Brick/Cement & 601 & 55.9 \\
\hline & Woods/Soil & 392 & 36.47 \\
\hline & Others & 82 & 7.63 \\
\hline \multirow{3}{*}{ Water Supply } & Pipe in the compound & 589 & 54.79 \\
\hline & Spring/Steam & 63 & 5.87 \\
\hline & Others & 17 & 1.58 \\
\hline \multirow{4}{*}{ Waste Disposal } & Latrine & 421 & 39.17 \\
\hline & Open Field & 507 & 47.16 \\
\hline & Municipality & 102 & 9.48 \\
\hline & Others & 45 & 4.19 \\
\hline \multirow{2}{*}{ Insects \& Rodents } & House flee & 345 & 32.1 \\
\hline & Others & 96 & 8.93 \\
\hline
\end{tabular}

\subsection{Maternal and Child Health Issues}

$341(68.47 \%)$ of the study participants were use FP. The commonest FP method used in Mettu town females was injectables which accounted 161 (47.13\%). Majority of women $81(51.59 \%)$ were bring forth their child at health institution.94 (59.87\%) women follow their ANC reguraly. Duration exclusive BF in majority of children $51(66.23 \%)$ were 6-24 months and continue complementary feeding < months were $43(55.84)$ and also only $42(54.54 \%)$ of children were fully vaccinated.

Table 4. Maternal and Child Health issues of respondents, Mettu town, 2021.

\begin{tabular}{llll}
\hline Variables & Category & Frequency & Percent \\
\hline \multirow{3}{*}{ Family Planning } & Pills & 52 & 15.29 \\
& Implants & 93 & 27.39 \\
& Injectables & 161 & 47.13 \\
Frequency of Antenatal & Others & 35 & 10.19 \\
& Reguraly & 94 & 59.87 \\
Site of Newborn & During Vaccination & 38 & 24.2 \\
& When Ill & 25 & 15.93 \\
Duration exclusive BF & Health Center & 81 & 51.59 \\
& Home & 76 & 48.41 \\
Complementary feeding & $<6$ months & 21 & 27.27 \\
& $6-24$ months & 51 & 66.23 \\
Immunization of $<5$ year Children & $>6$ months & 5 & 6.5 \\
& $<6$ months & 43 & 55.84 \\
& At 6 months & 21 & 27.27 \\
\end{tabular}




\subsection{The Disease Status in Mettu Town During Our Survey}

According to our study hypertension 263 (36.94\%) was most frequently encountered disease followed by diabetes mellitus $198(27.8 \%)$ in Mettu town population.

Table 5. The list of disease in mettu town during our study, Mettu town, 2021.

\begin{tabular}{|c|c|c|c|}
\hline Variables & Category & frequency & Percent \\
\hline \multirow{2}{*}{ Prevalence of health related problem } & Yes & 712 & 66.23 \\
\hline & No & 363 & 33.77 \\
\hline \multirow{4}{*}{ Type of disease } & Diabetes mellitus & 198 & 27.8 \\
\hline & Hypertension & 263 & 36.94 \\
\hline & Malaria & 109 & 15.32 \\
\hline & Others & 142 & 19.94 \\
\hline
\end{tabular}

\section{Discussion}

This survey was aimed to ascertain the apprehensive of health and health related problems. The age group 327 (30.4\%) of our respondents ranged from 19-35 years old. This is less than the studies employed in Bati Woreda, Oromia Zone, Amhara National Regional State which was 46.7\% [12]. This difference was due to age category. This age group is supposed to be the most productive and thus is susceptible to human health related problems especially women.

Our study shown that individuals, families, communities, and overall government expending are affected by the use of substances stirring the mind and behaviour. From those khat, alcohol, tobacco (cigarettes), had the rising prevalence calibers through all age groups which was comparable with the survey done in East Gojjam zone showed that, substance abuse such as high alcohol drinking, chat and Shisha were the push factors for early sex initiation to adolescents and youths [13].

Coinciding to our survey the houses of $55.9 \%$ respondents had a roof that was made of tin and floor made of cement and $36.47 \%$ individuals had a roof that was made of sheet and floor made of soil were good housing condition than the study done in East Gojjam zone showed that, all the studied houses had a roof that was made of sheet. $83.3 \%$ of studied houses had floor made of soil, and the rest $17.4 \%$ is made from cement [13]. This difference was due to economical and financial reason. As our study showed $54.79 \%$ of population use pipe water and $5.87 \%$ use spring/steam water. As the study done in Africa there were only 3 countries (congo, Mozambique, paupa new guinea) were less than half of the population have getting near to ameliorated water source. So the result of our survey is almost same with the study done in Africa [14]. 47.16\% of Mettu population use open field and $39.17 \%$ use pit latrine. When it compares it with the study done in Africa the sanitation practice improved over time and the coverage of improved sanitation is $64 \%$ and open defecation decreased to $14 \%$ and sub-Saharan African countries has made much slower progress [15]. The result showed that our country had unimproved use of sanitation because of Ethiopia is one of sub-Saharan country and the difference is also result of different study area and sample size and also there was time variation between our study and the study done in Africa. Rodent, household flee and bed bug are common insects \& rodents which whose consistent with the study done in East Gojjam which showed household fly, flea, mosquito, rodent (rat) and bed bug are common insects \&rodents [13]. These problems that seek peculiar concentration on the health of the community of the selected households because those insects or rodents perhaps threaten the health of population in oodles way.

Our survey showed that from $341(68.47 \%)$ user of family planning majority of them used $47.13 \%$ injection and $27.39 \%$ of them used implants and $31.53 \%$ respondents were used nothing. According to study done in Ethiopia overall $42 \%$ of females use family planning. Urban women are much likely to use their contraceptive than females in rural it is $50 \% \mathrm{Vs} 38 \%$ [16]. Our study showed that user of family planning were greater than the study done in Ethiopia because our study area was conducted solely in urban so urban area had stout variation when compared with the rural area and also because of difference in study population sample size.

Our survey showed that most of the women 81 (51.59\%) gave birth in health institution and 94 (59.87\%) attained ANC services at least one times reguraly was less than the study done in Nekemte City, Western Ethiopia which showed $93.04 \%$ of participants have attended ANC for their previous or last pregnancy [17]. This variation was due to site of study. In our study we ascertain the health related problem of general population when compared with study done in Nekemte City, Western Ethiopia which was solely conducted in new-born care practices and postnatal mothers. In our finding, fully immunized under 5 years children (54.54\%) coverage was lower than the survey employed in East Gojjam $60 \%$ [13]. This may be due to health extension workers did not bestow immunization service seemly, and low community apprehensive about immunization significance. In our survey, $27.27 \%$ of infants under 6 months are exclusively breastfed. Which was contradictory to recommendation by WHO those children under age 6 months should be exclusively breastfed, 17\% [18]. This shows the apprehensive of the community about exclusive breast feeding is meagre. According to our survey complementary feeding which showed, $55.84 \%$ of children start complementary feeding at 6 months while $16.89 \%$ of started complementary feeding at the age of $>6$ month was lower than the study carried out in Northern Ethiopia 
indicated at approximately $79.7 \%$ of mothers introduced complementary feeding at 6 months' age of the children as per recommended while $15.9 \%$ launched complementary feeding early before 6 months [7]. This shows the apprehensive of the community about complementary feeding is meagre.

According to our study the prevalence of health related problems was $712(66.23 \%)$, which was higher than the survey done in Amhara National Regional State (41\%) [12]. This indicates awareness of the community about health related problem is low. Hypertension $36.94 \%$ and diabetes mellitus $27.8 \%$ were the most common morbidity and mortality according to our study is consistent with the study conducted in Ethiopia which shows hypertension $18.8 \%$ and diabetes mellitus $27.9 \%$ are the two most common and easily diagnosed forms of NCDs [19]. Because the burden of NCDs was rising hastily amid lower income counter like Ethiopia.

\section{Conclusion and Recommendations}

The present study indicated that the prevalence of health related problem in individuals was high. Hypertension and diabetes mellitus are the grand cause of morbidity and mortality respectively. According our study majority of participants had a roof that was made of plate made of metal and floor made of cement. As our study shown majority of Mettu town population was use open field for waste disposal. In regard of using family planning many of females use injection followed by implants and one third of females were uses nothing. The practices of exclusively breastfed and timely initiation of complementary feeding in our survey was low due to awareness of the community about exclusively breastfed and complementary feeding was meagre. Health professionals should have to give the population psycho education about substance, waste disposal, family planning, sanitation condition, immunization and Mettu town administration should have to prepare waste disposal places for proper waste disposal in the town.

\section{Abbreviations}

AIDS: acquired immuno deficiency syndrome; ANC: Ante natal Care; BF: Breast feeding; CBE: Community based education; CBTP: Community Based Training Program; DTTP: Development Team Training Program; FP: Family planning; HIV: Human immune deficiency virus; NCD: Non Communicable Disease; SRP: Student Research Program; STI: Sexually Transmitted Infections; TB: Tuberculosis; TTP: Team Training Program.

\section{Acknowledgements}

We would like to explicit our profound and unfeigned appreciation to all data collectors and respondents for their through going time they bring forth us.

\section{References}

[1] Department of Health and Human Service, North Carolina (2002). Community Assessment Guide book: www.healathcarolinians.org.

[2] Faris K, Kaba M. Hygienic behaviour and environmental conditions in Jimma Town, South-western Ethiopia. The Ethiopian Journal of Health Development (EJHD). 2017. 13 (2). Retrieved from https://ejhd.org/index.php/ejhd/article/view/897.

[3] WaSH Ethiopia: Sanitation Coverage, WHO Communicable disease prevention, control and eradication, 2010.

[4] Whiteford HA, Degenhardt L, Rehm J, Baxter AJ, Ferrari AJ, Erskine HE, et al. Global burden of disease attributable to mental and substance use disorders: findings from the Global Burden of Disease Study 2010. Lancet (London, England). 2013; 382 (9904): 1575-86. Epub 2013/09/03. doi: 10. 1016/s0140-6736(13)61611-6. PubMed PMID: 23993280.

[5] UNICEF, Southern African region and the response of education systems to HIV/AIDS: Life skills programs, 1999.

[6] Abebaw D. Determinants of solid waste disposal practices in urban areas of Ethiopia: a household-level analysis. Eastern Africa Social Science Research Review. 2008; 24 (1): 1-14.

[7] Central Statistical Agency (CSA) [Ethiopia] and ICF. 2016. Ethiopia Demographic and Health Survey 2016. Addis Ababa, Ethiopia, and Rockville, Maryland, USA: CSA and ICF.

[8] Ayenew AA, Nigussie A, Zewdu B. Prevalence of home delivery and associated factors in Ethiopia: A systematic review and meta-analysis. 2020.

[9] Van Damme H. Domestic water supply, hygiene, and sanitation. Overcoming Water Scarcity and Quality Constraints IFPRI 2020 Focus 9, Brief. 2001; 3.

[10] Chaka EE, Abdurahman AA, Nedjat S, Majdzadeh R. Utilization and determinants of postnatal Care Services in Ethiopia: a systematic review and meta-analysis. Ethiopian journal of health sciences. 2019; 29 (1). DOI: 10.4314/9ejhs.v29i1.16.

[11] Demissie T, Kogi-Makau W. Food taboos among pregnant women in Hadiya Zone, Ethiopia. The Ethiopian Journal of Health Development (EJHD). 1998; 12 (1) (ISSN1021-6).

[12] Yonas B, Kumie a. Assessment of health hazards and associated factors among the returned migrants living at Bati Woreda, Oromia Zone, Amhara National Regional State. Ethiop. J. Health Dev. 2013; 27 (1): 55-63.

[13] Gelaw BK, Tegegne GT, Bizuye YA (2014) Assessment of Community Health and Health Related Problems in Debre Markos Town, East Gojjam, Ethiopia, 2013. J Biosafety Health Educ 2: 125. doi: 10.4172/2332-0893.1000125.

[14] Beyene M, Worku AG, Wassie MM. Dietary diversity, meal frequency and associated factors among infant and young children in Northwest Ethiopia: a cross-sectional study. BMC public health. 2015; 15 (1): 1007.

[15] Erasu D, Feye T, Kiros A, Balew A. Municipal solid waste generation and disposal in Robe town, Ethiopia. Journal of the Air \& Waste Management Association. 2018; 68 (12): 1391-7. 
[16] Central Statistical Agency (CSA) [Ethiopia] and ICF. 2019. Ethiopia mini Demographic and Health Survey 2019. Addis Ababa, Ethiopia, and Rockville, Maryland, USA: CSA and ICF.

[17] Efa BW, Berhanie E, Desta KW, Hinkosa L, Fetensa G, Etafa $\mathrm{W}$, et al. (2020) Essential newborn care practices and associated factors among postnatal mothers in Nekemte City, Western, Ethiopia. PLoSONE15 (4): e0231354. https://doi.org/10.1371/journal.pone.0231354.
[18] Shumey A, Demissie M, Berhane Y. Timely initiation of complementary feeding and associated factors among children aged 6 to 12 months in Northern Ethiopia: an institution-based cross-sectional study. BMC public health. 2013; 13 (1): 1050.

[19] Abera SF, Gebru AA, Biesalski HK, Ejeta G, Wienke A, Scherbaum V, et al. Social determinants of adult mortality from non -communicable diseases in northern Ethiopia, 2009-2015: Evidence from health and demographic surveillance site. PloS one. 2017; 12 (12): e0188968. 